\title{
RETOS, PROFESORES Y ALUMNOS CON TALENTO MATEMÁTICO
}

\section{Challenges, teachers and gifted learners in mathematics}

\author{
Enrique Castro, Juan F. Ruiz-Hidalgo y Elena Castro-Rodríguez \\ Universidad de Granada \\ Correo-e: ecastro@ugr.es; jfruiz@ugr.es; elenacastro@ugr.es \\ Recepción: 6 de octubre de 2014 \\ Envío a informantes: 7 de octubre de 2014 \\ Aceptación definitiva: 18 de diciembre de 2014 \\ Biblid. [0214-3402 (2015) (II época) n. ${ }^{\circ}$ 21; 85-104]
}

Resumen: En este artículo reflexionamos sobre la naturaleza de los retos y de su importante papel en la atención de los estudiantes con talento matemático e incidimos en las posibles funciones que puede desempeñar el profesor. Destacamos la necesidad de que el profesor de matemáticas tenga un conocimiento especializado sobre la formulación de retos matemáticos apropiados para la identificación y el estímulo de los estudiantes con talento matemático en el aula. Ejemplificamos estas ideas con retos específicos, mostrando estrategias que puede utilizar el profesor para formular retos matemáticos en torno a un contenido específico y cómo organizar su secuencia didáctica en el aula.

Palabras Clave: estudiantes con talento matemático; retos matemáticos; talento matemático; profesores; reformulación de tareas.

AвSTRACT: In this paper we reflect on the nature of the mathematical challenges and their important role in the care of students with mathematically talented students, and we insist on the possible roles that teachers can play. We stress the need for the math teacher achieve a specialized knowledge on formulating appropriate mathematical challenges, in order to identify and to encourage mathematically talented students in the classroom. We exemplify these ideas with specific challenges, showing teacher strategies used to formulate mathematical challenges around a specific content and how to sequence them.

KEY WORDS: mathematically talented students; mathematical challenges; mathematical talent; teachers; reformulation of task. 
RETOS, PROFESORES Y ALUMNOS CON TALENTO MATEMÁTICO

ENRIQUE CASTRO, JUAN F. RUIZ-HIDALGO Y ELENA CASTRO-RODRÍGUEZ

\section{Introducción}

$\mathrm{S}$ ALVO EN ACTIVIDADES DE ENRIQUECIMIENTO PUNTUALES, como cursos de verano o programas extraescolares de estímulo del talento, los alumnos con alta capacidad para las matemáticas suelen estudiar en clases heterogéneas en las que siguen el programa oficial establecido y no reciben trato especial. Hay anécdotas que subrayan la falta de atención en el aula a este colectivo de estudiantes. Refiramos como ejemplo la anécdota hipotética de un padre que tiene escolarizado a un niño con altas capacidades:

Tengo un niño de altas capacidades que se aburre en clase.

He hablado con el maestro y le he expuesto mi preocupación.

El maestro me replica:

No puedo dedicarme a su hijo. Tengo que desarrollar el programa.

La anécdota tiene visos de realidad y puede haberse repetido con relativa frecuencia en el ámbito escolar. En su respuesta, el maestro justifica la situación por el imperativo oficial de desarrollar todo el programa, que puede ocultar otras carencias que sí podrían abordarse. El aburrimiento en la escuela es una de las principales problemáticas relativas a todos los alumnos y que afecta también a los estudiantes con altas capacidades (Feldhusen y Kroll, I99I). Walters y Diezmann (2000), citando trabajos previos, subrayan como una de las razones el tipo de tareas académicas que se desarrollan en el aula, que no suponen un desafío para los estudiantes más capaces. También destacan estos autores que los estudiantes con talento otorgan poco valor a las actividades de aprendizaje que realizan en el aula y no las afrontan como un reto, lo que es un hándicap para su aprendizaje.

A este respecto, Miller (1990) subraya que estudiantes con talento pueden pasar inadvertidos para el profesor y no ser atendidos adecuadamente en el sistema escolar.

Algunos estudiantes con talento matemático no demuestran logros académicos, no muestran entusiasmo hacia los programas de matemáticas de la escuela, ni a conseguir las mejores calificaciones en la clase de matemáticas. Es importante saber que hay estudiantes de este tipo, y que su habilidad para las matemáticas se pasa por alto con facilidad, a pesar de que pueden presentar otros indicios que sugieren alta capacidad en las matemáticas (Miller, 1990).

Miller subraya la importancia que tiene en este hecho el programa escolar de matemáticas, que en definitiva es la importancia de las tareas matemáticas que se les proponen a estos estudiantes. Asimismo, señala la falta de preparación de los profesores para detectar alumnos con talento y para ajustar los programas a las características de estos estudiantes con más capacidades.

Para paliar la falta de concordancia entre los programas escolares y las necesidades de los estudiantes más capaces, se han realizado propuestas como la de Johnson (1994), en las que se contempla que el currículo para los alumnos con talento matemático debería ser modificado de tal manera que las tareas matemáticas que se les proponen a diario estuvieran de acuerdo a sus capacidades. Esta idea debería aplicarse a todos los estudiantes en general, pero en la práctica se atiende a un sujeto ideal medio al que se dirigen las actividades. Johnson (I994) recomienda elementos clave que se deben tener en cuenta cuando se elabora un currículo para estudiantes con talento matemático: 
- El alcance del contenido debe ser lo más amplio posible.

- El contenido debería presentárseles con una gran profundidad y con un alto grado de complejidad, combinado con conceptos en forma abstracta.

- El currículo debería presentarse como una actividad de descubrimiento guiado que permita la exploración de conceptos.

- La instrucción debería centrarse en resolución de problemas.

- Los profesores deberían emplear un enfoque metacognitivo en el que los estudiantes se centren en los procesos de resolución de problemas.

- Las matemáticas deberían estar conectadas con otras disciplinas. Por ejemplo, con las ciencias o la música.

Sheffield (1999) subraya al respecto que los programas para los estudiantes con talento matemático deberían no sólo incrementar la aceleración del currículo ofrecido, deberían también contemplar mayor profundidad y ayudar a los estudiantes a ver las conexiones entre los conceptos matemáticos. En cuanto a los contenidos, Assouline y Lupkowski-Shoplik (2005) precisan que los alumnos con talento matemático deberían estudiar una variedad de tópicos que incluyen los habituales: resolución de problemas, geometría, álgebra, aritmética, sistemas numéricos, estadística, probabilidad y visualización espacial. La clave de la adaptación de los currículos a las necesidades de los estudiantes con talento es enriquecer el currículo de manera sistemática. Para ello, Assouline y Lupkowski-Shoplik (2005) proponen como tópicos interesantes para enriquecer el currículo los referidos a polígonos estrellados, fractales, teorema de Pitágoras y ternas pitagóricas, números de Fibonacci, razón áurea, cuadrados mágicos, sistemas de numeración antiguos, números figurados y topología. Estos tópicos permiten trabajar en el aula tareas estimulantes que supongan un reto para los estudiantes y potencian pensamiento matemático más avanzado que el correspondiente al nivel medio de la clase.

\section{Retos matemáticos}

Las tareas académicas constituyen el trabajo del aula y, supuestamente, proporcionan el desafío suficiente para que aflore el aprendizaje (Doyle, 1988). La naturaleza de la tarea juega un papel crítico en la determinación del tipo de comprensión matemática que el estudiante alcanzará en la clase de matemáticas (Hiebert et al., 1997). Las tareas ricas deben ir más allá de los problemas de cálculo (Lampert, 1990) y deben permitir a los estudiantes llevar a cabo una comprensión de los conceptos matemáticos que integre tanto el conocimiento conceptual como el procedimental.

En la literatura especializada sobre educación matemática no hay una aceptación universal de términos, tales como tarea, ejercicio, problema, ni tampoco la hay del término reto cuando nos referimos a tareas escolares o problemas (Powell et al., 2009), por ello, clarificaremos el significado que le otorgamos a estos términos. Desde un punto amplio en Barbeau y Taylor (2009) se considera un reto a una cuestión planteada deliberadamente para atraer a un estudiante a que intente obtener una respuesta, ampliando al mismo tiempo su comprensión y conocimiento de un tema. La cuestión de si es un reto o no depende del estudiante: lo que puede ser un rompecabezas para una persona puede ser un ejercicio rutinario o una cuestión de recordar para otra con 
más experiencia. Un reto puede o puede no ser apropiado. Un reto es inapropiado si el destinatario no tiene la formación adecuada para entender lo que está en juego o no posee o no puede disponer de las herramientas necesarias para abordarlo. Un buen reto es aquel para el cual la persona posee el aparato matemático o las destrezas lógicas necesarias, pero necesita emplearlas de forma original e innovadora.

Las ideas anteriores enlazan con la opinión de expertos de varios países cuando subrayan que se deberían potenciar las cualidades de los alumnos con talento matemático (creatividad, curiosidad, originalidad, persistencia y flexibilidad) a partir de tareas matemáticas de resolución de problemas en el sentido clásico del término (Charles y Lester, I982; Polya, I973; Schoenfeld, I985), de tal manera que las tareas o problemas que aborden les supongan un verdadero reto (Applebaum y Leikin, 2007; Freiman, 2006; Sheffield, 2003).

En adelante, entenderemos por tarea matemática escolar una demanda estructurada de actuación que el profesor proporciona a los estudiantes. Las tareas pueden ser ejercicios rutinarios y problemas. La distinción entre ejercicio y problema no se puede realizar sin tener en cuenta al estudiante. Una tarea matemática es un ejercicio para un estudiante si ya tiene experiencia, si conoce el proceso que hay que seguir para realizarla, porque lo ha aprendido o practicado previamente. Por el contrario, si la tarea no es rutinaria porque el estudiante no tiene experiencia previa, o no conoce de antemano el camino a seguir para realizar la tarea, está ante un problema. En este sentido y a los efectos de este estudio, identificamos reto con problema. Los profesores deben ser cuidadosos en las tareas que seleccionan como retos. Leikin (2004) considera que los retos matemáticos que se les propongan a los estudiantes con talento deben cumplir una serie de condiciones: a) ser motivadores; b) que no se resuelvan fácilmente con procedimientos disponibles; c) que requieran que el estudiante realice un intento; d) tener varios enfoques para obtener la solución. Obviamente, advierte Leikin (2004), estos criterios son relativos a la experiencia que tenga el estudiante en el campo, puesto que una tarea puede ser difícil para unos estudiantes y trivial para otros.

\section{Finalidades de los retos}

Los retos matemáticos se pueden utilizar con dos finalidades en la educación de los escolares. Por un lado los retos matemáticos se pueden utilizar para un diagnóstico inicial del talento matemático de los estudiantes y, por otro, se pueden emplear durante el proceso de intervención para enriquecer y potenciar las dotes intelectuales de los alumnos. Pero en un mismo nivel educativo lo que es un reto para un estudiante puede no serlo para otro, por lo que hay que seleccionar los retos de acuerdo a las capacidades de los estudiantes si queremos que cumplan su función de desafío intelectual.

\section{I. Retos para identificar a estudiantes con talento}

El proceso de identificación de niños con talento matemático supone el empleo de métodos cualitativos y cuantitativos de manera complementaria. Pero es importante que los profesores conozcan que los indicadores de los que disponemos y que 
nos proporcionan los diversos instrumentos que se suelen aplicar en la identificación no todos tienen el mismo valor. Investigaciones como las de Ellerton (1986), Krutetskii (1969), Niederer e Irwin (200I), Niederer, Irwin, Irwin y Reilly (2003) y Span y Overtoom-Corsmit (1986) han destacado que los indicadores de talento matemático procedentes de instrumentos que emplean problemas matemáticos (no rutinarios) son un método eficaz para caracterizar el talento matemático.

En el trabajo de Pasarín, Feijoo, Díaz y Rodríguez (2004) se subraya «la baja relación existente entre los tests utilizados para evaluar la aptitud matemática y las características fundamentales del talento matemático» (p. 99) destacadas por Greenes (198I). Como consecuencia los autores afirman que este divorcio señala la necesidad de incluir problemas específicos (que denominaremos retos) para una detección más correcta de la aptitud matemática. Nos dan como ejemplo representativo de tarea que puede considerarse un reto para alumnos de $2 .^{\circ}$ de secundaria el siguiente problema:

\section{Problema n. ${ }^{\circ}$-tostado rápido}

Hay que tostar en una parrilla tres rebanadas de pan. En la parrilla caben dos rebanadas de pan a la vez, pero sólo se pueden tostar por un lado. Se tardan 30 segundos en tostar una cara de una pieza de pan, s segundos en colocar una rebanada o en sacarla, y 3 segundos en darle la vuelta. ¿Cuál es el mínimo tiempo que se necesita para tostar las tres rebanadas?

(Pasarín y otros, 2004: 90)

Uno de los identificadores más utilizado para considerar que un estudiante tiene talento matemático son sus calificaciones en la asignatura. Pero hay que tomarlo con cautela, pues en ellas se tienen en cuenta aspectos que no corresponden al talento matemático. Muchos alumnos con talento no obtienen buenas calificaciones. Las calificaciones finales que se otorgan a los estudiantes en la asignatura de matemáticas pueden ser un falso indicador de talento matemático o un indicador menos fiable que una prueba de resolución de problemas donde se ponga a prueba su razonamiento (Castro, Benavides y Segovia, 2006).

\subsection{Los retos como fuente de aprendizaje}

Desde el punto de vista educativo, Watters y Diezmann (200o) afirman que todos los estudiantes necesitan tareas desafiantes para facilitar el aprendizaje y desarrollar su autonomía personal. Estos autores dan razones de carácter cognitivo, metacognitivo y afectivo para que se utilicen retos matemáticos en la formación de los estudiantes con talento. En primer lugar los retos matemáticos facilitan el desarrollo de la cognición puesto que proporcionan una base para el desarrollo del potencial matemático de los estudiantes. Con ellos los estudiantes ponen en juego pensamiento matemático avanzado, razonamientos complejos, exploran patrones y relaciones, trabajan de forma abstracta y pueden producir soluciones originales y creativas. En segundo lugar los retos matemáticos pueden contribuir a que los estudiantes con 
talento utilicen y desarrollen destrezas metacognitivas. Conocer qué conocimiento emplear en cada momento, saber seleccionar la estrategia apropiada para resolver un problema o rechazar una por inapropiada y evaluar la razonabilidad de una respuesta dada a un problema forman parte del tipo de conocimiento que es útil en el quehacer de un matemático. Por último, los retos matemáticos hacen emerger en los estudiantes aspectos afectivos favorables para el proceso de aprendizaje. Con ellos, los estudiantes se muestran más predispuestos y motivados para realizar las tareas y, por tanto, para alcanzar un mayor desempeño en matemáticas. El ser capaces de abordar tareas desafiantes contribuye a que suba su autoestima y la confianza en sus capacidades.

\section{Funciones del profesor}

El profesor desempeña funciones clave en la atención a los estudiantes con más capacidad. Entre ellas está seleccionar tareas de dificultad apropiada para el nivel de los estudiantes (Henningsen y Stein, 1997). Si es necesario, el profesor tiene que moderar la dificultad de las tareas para determinados estudiantes debido a que la misma tarea puede no ser de valor equivalente para la diversidad de estudiantes presentes en el aula. También debe facilitar la cognición de alto nivel, para ello, el maestro tiene que «de manera proactiva y consistente dar apoyo a la actividad cognoscitiva de los estudiantes, sin reducir la complejidad y la demanda cognitiva de la tarea» (Henningsen y Stein, 1997: 546). El profesor puede proporcionar extrínseca e intrínsecamente apoyo a los estudiantes mediante la participación en la práctica del aprendizaje cognitivo, que es un conjunto de estrategias de enseñanza para el desarrollo de conocimientos en ámbitos como las matemáticas (Collins, Brown y Newman, 1989).

Cuando el maestro plantea una tarea en un aula en la que aprenden juntos alumnos con diferentes capacidades, debe ser consciente de que será un reto para algunos de ellos, pero que puede que no lo sea para otros. Para cumplir las expectativas de que las tareas supongan un reto para la diversidad de alumnos en el aula, puede optar por plantear tareas que sean adaptativas y que puedan reformularse con distintos niveles de dificultad. La reformulación de problemas es una destreza profesional que deberían adquirir los maestros y profesores de matemáticas. Pero, ¿de qué modo y bajo qué supuestos se debe actuar en la reformulación de un problema de matemáticas para que les plantee un reto a alumnos con talento matemático?

\section{Reformulación de tareas}

El profesor de matemáticas de Educación Primaria puede elaborar retos para sus alumnos a partir de los problemas rutinarios que emplea en clase. Para ello puede utilizar la técnica de reformular problemas y, a partir de un problema dado, realizar algún cambio o cambios en alguna de sus características que lo conviertan en un problema más difícil de resolver. En este caso el profesor debe tener en mente si el problema resultante de la reformulación pone en juego al menos una de las características detectadas en los estudiantes con talento matemático (Greenes, I98I; Krutestskii, I976; Miller, I990). 


\section{I. Reformulación de tareas. Modificar datos}

Centrándonos en el nivel de primaria, los problemas aritméticos aditivos que se utilizan suelen corresponder a alguna de las categorías semánticas establecidas (cambio, combinación o comparación) y según el nivel escolar hay diferencia de dificultad entre ellos. Las categorías más difíciles puede utilizarlas el profesor con alumnos más capaces. Así mismo, si consideramos, por ejemplo, los problemas aritméticos simples de un paso de la categoría semántica de comparación multiplicativa, en los que intervienen tres cantidades: el referente, el comparado y el escalar que cuantifica la comparación, resulta que en los curso $5 .^{\circ}$ y $6 .^{\circ}$ de primaria las diferencias de dificultad entre estos tres problemas son notorias (Castro, I995).

TABla I. Índices de dificultad globales de los problemas aritméticos de comparación multiplicativa (Castro, 1995)

\begin{tabular}{|c|c|c|c|}
\hline $\begin{array}{r}\text { Índice de } \\
\text { dificultad }\end{array}$ & $\begin{array}{c}\text { Comparado } \\
\text { desconocido }\end{array}$ & $\begin{array}{c}\text { Escalar } \\
\text { desconocido }\end{array}$ & $\begin{array}{c}\text { Referente } \\
\text { desconocido }\end{array}$ \\
\hline $5 .^{\circ}$ de primaria & 0,685 & 0,284 & 0,497 \\
\hline $6 .^{\circ}$ de primaria & 0,802 & 0,460 & 0,498 \\
\hline
\end{tabular}

Como puede observarse en los índices de dificultad (véase Tabla i) en los problemas de referente desconocido, tanto en $5 .^{\circ}$ como en $6 .^{\circ}$ curso, el porcentaje de alumnos que los resuelven no llega al 50\% de la clase. En una investigación posterior empleando como muestra a sujetos con talento de la misma edad, el porcentaje de aciertos llegó al 90,8\%. Por lo que está claro que esta forma de reformular problemas hace poner en juego una característica del talento matemático. En niveles inferiores de primaria, por ejemplo, en $4 .^{\circ}$, su efecto sobre la detección de esta característica del talento matemático es más pronunciada, reduciéndose el porcentaje de aciertos a un grupo de estudiantes más capaces. En este caso el profesor puede formular un problema de comparación de referente desconocido a partir de un problema de multiplicación de comparado desconocido, lo que obliga al estudiante a poner en práctica su habilidad para invertir relaciones

\subsection{Reformulación de tareas. Cambiar la naturaleza de los números}

Otra forma de obtener problemas que les planteen una cierta dificultad a los alumnos con talento es cambiando la naturaleza de los números. Para los alumnos de quinto y sexto curso de primaria el problema A tiene un índice de dificultad muy bajo.

Problema A. Una pieza de cobre pesa 9 kilogramos. Si un kilogramo cuesta 6 euros, ¿cuánto cuesta la pieza de cobre? (I00\% de éxito).

Si sustituimos los números naturales por números decimales el problema se convierte en un problema con una dificultad alta para los estudiantes de estos niveles, y 
si uno de los números decimales es menor que uno la dificultad es aún mayor. En la investigación de Benavides (2008) con estudiantes de quinto y sexto curso de primaria, se emplean dos problemas derivados del anterior, que denominamos problema $\mathrm{B}$ y problema C.

Problema B. Un trozo de queso pesa 0,923 kilogramos. Si un kilo cuesta 6,50 euros, ¿cuánto cuesta el trozo de queso? (40\% de éxito).

El problema B tuvo un $40 \%$ de éxito entre los estudiantes con talento, pero este éxito se debió en parte a que hubo estudiantes que utilizaron estrategias originales. Por ejemplo, uno de los estudiantes da la siguiente explicación: primero en el apartado he calculado lo que cuestan ioo gramos, para ello, divido 6,5 entre io. El resultado se lo resto a 6,5 y me da lo que valen 900 gramos que son 5,85 . Me falta por calcular lo que valen los 23 gramos. Puesto que 23 es aproximadamente la cuarta parte de Ioo, en el apartado c divido o,65 que es lo que valen ioo gramos entre 4, me da 0,1625. Se lo sumo a lo que valen los 900 gramos (apartado d) y me da lo que vale el trozo de queso, aunque no es exacto porque la cuarta parte de Ioo es 25 no 23.

Está claro que este estudiante con talento no asocia el problema con realizar una multiplicación. Para él no es un problema rutinario. Emplea sus conocimientos aritméticos de manera flexible dando lugar a una estrategia original que no queda empañada por el error de cálculo final.

El estudiante anterior emplea la idea de aproximación de forma razonable al igual que otro estudiante con talento de la misma edad que ante el problema $\mathrm{C}$ establece cinco pasos para la resolución que además enumera.

Problema C. Si o,923 kilogramos cuestan 6 euros. ¿Cuánto cuesta el kilo de queso? ( $37 \%$ de éxito).

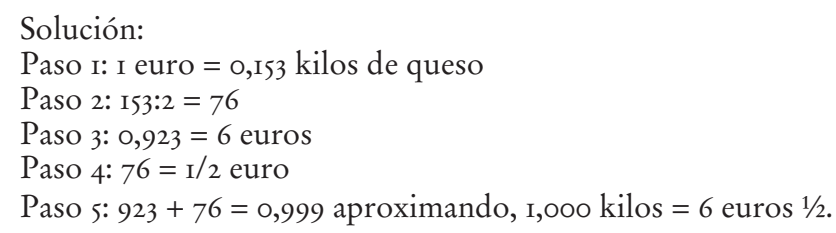

Para estos estudiantes, tanto el problema B como el C han sido verdaderos retos, que les han obligado a poner en juego razonamientos numéricos complejos para su nivel de desarrollo, lo que evidencia que posee un sentido numérico avanzado para su edad.

\subsection{Reformulación de tareas. Modificar condiciones}

Cuando los estudiantes con talento matemático se encuentran ante una tarea que consideran fácil suelen ir más allá de lo que se demanda en ella, o pueden plantearse preguntas adicionales. Por ejemplo, pueden realizar un proceso de generalización de modo espontáneo en tareas que sólo tratan casos específicos o pueden estar interesados en saber qué pasa cuando se modifican alguna de las condiciones de una tarea. El profesor puede aprovechar esta cualidad de los estudiantes con talento para plantearles retos que van más allá de lo que se exige en el currículo. 
Los estudiantes con talento aprenden con gran rapidez y no necesitan tanta práctica para dominar destrezas y procedimientos matemáticos. Si el profesor les induce a realizar el mismo número de prácticas que al resto de la clase surge el problema de encontrarse desocupado o de aburrirse. Para ello, el profesor puede plantearle retos adicionales ligados con el tópico escolar que se está trabajando en clase y que suponen un enriquecimiento conceptual para los estudiantes. Uno de los tópicos mencionados por Assouline y Lupkowski-Shoplik (2005) como apropiado para desarrollar el talento matemático es el teorema de Pitágoras y las ternas pitagóricas. Entre las opciones que tiene el profesor sobre este tópico para formular un reto para sus alumnos más capaces citemos la siguiente, que surge de cambiar una de las condiciones en el enunciado del teorema expresado como igualdad de áreas.

En un triángulo rectángulo, el área del cuadrado construido sobre la hipotenusa es igual a la suma de las áreas de los cuadrados construidos sobre los catetos.

Ante una visualización geométrica como ésta del teorema de Pitágoras surge la pregunta de si sustituyendo la figura del cuadrado por un triángulo equilátero se sigue cumpliendo la relación entre las áreas, es decir, si es cierto que:

En un triángulo rectángulo, el área del triángulo equilátero construido sobre la hipotenusa es igual a la suma de las áreas de los triángulos equiláteros construidos sobre los catetos.

Figura i. Teorema de Pitágoras

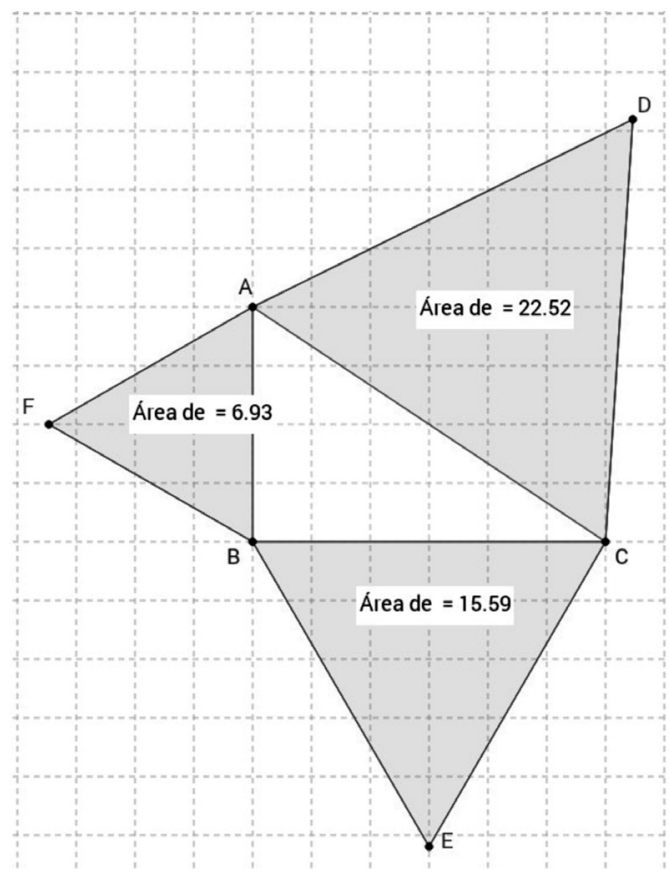


El reto se puede continuar empleando otras figuras geométricas regulares como círculos o semicírculos, lo que proporciona mayor generalidad a la expresión, y examinando las condición de regularidad con figuras no regulares, para ver si la regularidad es una condición que deben cumplir las figuras para que la relación se mantenga.

\subsection{Reformulación de tareas: retos análogos}

Consideramos que dos retos son análogos cuando son isomorfos matemáticamente, difieren sólo en el contexto o situación real sobre la que se plasman las relaciones matemáticas y, por tanto, los estudiantes los pueden resolver empleando los mismos conceptos y procedimientos matemáticos en el mismo nivel escolar. El profesor debe gestionar la oportunidad de emplear uno u otro reto análogo en función del momento didáctico que viva con sus alumnos.

Los tres problemas siguientes que hemos denominado problema de las pizzas, problema de las torres y problema de las luces, son ejemplo de problemas análogos.

Problema de las pizzas. Una pizzería vende pizzas a gusto del consumidor. Partiendo de una base de pizza con salsa de tomate, el cliente puede añadirle los siguientes ingredientes: pimientos, salchichas, champiñones y pepperoni. ¿Cuántas elecciones pueden realizar los clientes? Encuentra la manera de convencer a otro de que has encontrado todas las posibilidades.

Problema de las torres. Con cubos Unifix de dos colores queremos formas torres de cinco pisos. Si consideramos que una torre difiere de otra por el color, ¿cuántas torres distintas podemos formar?

Problema de las luces. En una oficina de la ciudad identifican a los empleados mediante luces intermitentes. Cada empleado tiene una combinación consistente en una o más luces. Existen exactamente tantas combinaciones como empleados. Hay cinco luces de colores en un panel como el siguiente, ¿Cuántos empleados hay en la oficina?

Figura 2. Problema de las luces

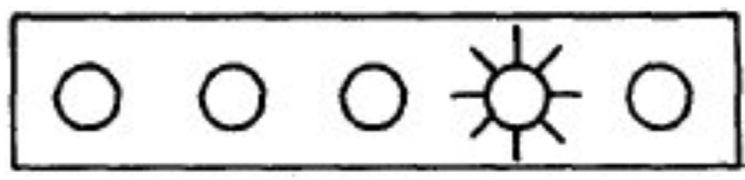

Los tres problemas están conectados por su estructura matemática y contribuyen cada uno en un aspecto a consolidar en los estudiantes el esquema multiplicativo. Abundando en esta idea, el profesor puede construir nuevos retos planteando problemas análogos a partir de uno dado. En una prueba de identificación o en un proceso de intervención pueden sustituirse uno de ellos por otro, desempeñando cualquiera de ellos el mismo papel y el nivel de reto matemático que los otros. 
El profesor debe ser capaz de identificar estos problemas como análogos, pero, además, ¿qué puede esperar un docente de matemáticas que respondan sus alumnos con talento matemático? ¿A qué niveles escolares es adecuado plantearse aplicarlo? ¿Para qué fines de aprendizaje matemático pueden utilizarse estas tareas? Para contestar a estas preguntas es conveniente realizar un análisis cognitivo de las tareas. Para ejemplificar el análisis cognitivo vamos a analizar el problema de las luces.

\section{Conocimiento cognitivo de la tarea}

Cuando se utilizan problemas matemáticos como retos es útil que el docente tenga conocimiento de las dificultades que esa tarea conlleva para alumnos de un determinado nivel de desarrollo, así como de las posibles estrategias con las que se espera que los estudiantes aborden su realización. También tiene que estar preparado el profesor para asumir que una estrategia no usual empleada por el estudiante en la solución de un problema puede ser adecuada, y estar dispuesto a examinar a fondo estas soluciones no usuales. El conocimiento que se puede obtener mediante un análisis cognitivo a priori de la tarea puede ayudar al profesor a controlar su dificultad para los estudiantes, el tipo de estrategias que es previsible que utilicen o los interrogantes que puedan plantear.

Fijémonos como ejemplo en qué podría ser un análisis cognitivo a priori del problema de las luces, que podría extenderse a los problemas análogos.

Hay datos suficientes con estudiantes de entre io y i4 años de edad en los que se pone de manifiesto cómo van aflorando distintas estrategias de resolución según el nivel de desarrollo de los estudiantes. Niederer e Irwin (200I) aplican este problema a siete escolares de diez años de edad que habían sido ya identificados como niños con talento matemático. Sólo uno de los escolares identificado como sujeto con talento obtuvo la respuesta correcta. Concretamente escribe como solución:

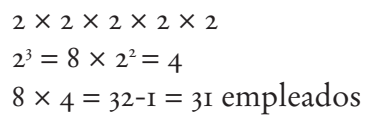

El escolar ha reconocido la naturaleza del problema y utiliza procedimientos matemáticos apropiados para obtener la solución. Para Niederer e Irwin (200I) esta solución es propia de un alumno con altas capacidades en el sentido que le da Krutestkii (1976) a este término. Este trabajo nos da un indicio de que la regla del producto, tal como se entiende en combinatoria, la aplican los sujetos con talento de manera espontánea, sin haber recibido instrucción previa.

Una segunda estrategia que aflora de manera espontánea en los sujetos es realizar combinaciones. Niederer e Irwin (200I) comparan esta solución con la dada por otro niño identificado con altas capacidades mediante test de inteligencia y que tiene una puntuación similar en el test. En esta segunda respuesta, el estudiante no comprende el problema y emplea las iniciales de los colores en inglés para etiquetar las luces y efectuar combinaciones con ellas. Utiliza la estrategia de listar todas las posibilidades. Propone sus primeras cinco soluciones ignorando que las luces de colores no pueden cambiar de posición (Tabla 2). 
TABLA 2. Cinco primeras soluciones del problema Niederer e Irwin (200I)

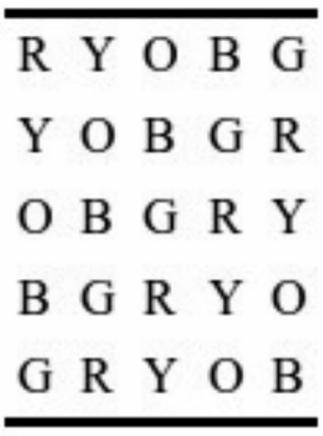

Realiza combinaciones similares con cuatro, tres, dos y una luz, dando como respuesta 25. Los resultados de este trabajo nos hacen pensar que formar combinaciones es un proceso complicado para los sujetos con talento de esta edad.

Al margen de que el color de las luces actúa como un distractor, el problema de las luces es demasiado difícil para los estudiantes de tercer y cuarto curso de primaria. La pregunta que nos hacemos es cómo evolucionará esto en los años sucesivos antes de que se explique la combinatoria oficialmente en el currículo escolar que es en $4 .^{\circ}$ de la Eso.

En un estudio posterior en el que participan niños con talento matemático de edad comprendida entre in y i2 años de edad de Santiago de Chile (Benavides, 2008) se utiliza el problema de las luces pero eliminando el distractor de que las luces sean de color (Span y Overtoom-Corsmit, 1986), el problema formaba parte de un cuestionario de problemas. Este problema resultó ser difícil para los niños con talento. Cuatro de los 30 niños con talento dieron como solución el producto de $2 \times 2 \times 2 \times 2 \times 2$, obviando que la combinación de las cinco luces apagadas no contaba como se puede ver en la Figura 3.

Figura 3. Regla del producto empleada por un estudiante
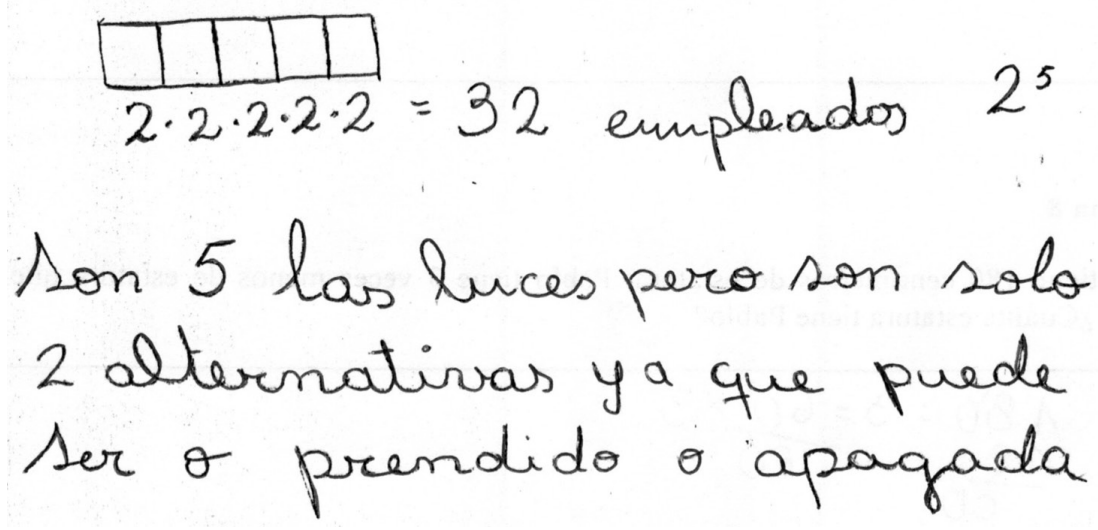

Al igual que en el caso de Niederer e Irwin (200I) los sujetos que enfocan la solución de tal manera que les puede llevar a la solución correcta emplean la regla del producto 
de las posibilidades de cada luz (véase Figura 4) y, en el resto de las respuestas, no aparece utilizada correctamente la idea de combinación.

Uno de los sujetos en este estudio da como respuesta $4 \times 5=20$, interpretando que sólo se puedan dar combinaciones de luces de dos en dos. Con cada luz se pueden formar cuatro parejas, luego el total es $4 \times 5=20$.

Figura 4. Respuesta dada por un estudiante $4 \times 5=20$
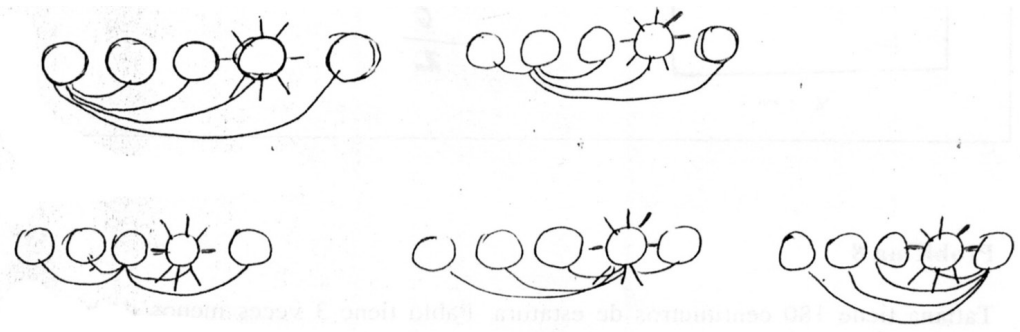

$4 \times 5=20$

R/ hay 20 Empleados en le oficima

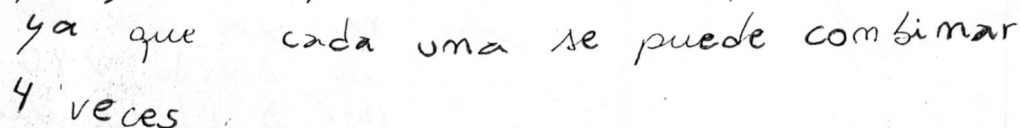

Además de utilizar sólo parejas, otro estudiante impone la restricción de formarlas de manera ordenada, lo que le sale la suma $4+3+2+\mathrm{I}=$ Io. Con ello evita las soluciones duplicadas.

FIgURA 5. Respuesta dada por un estudiante $4+3+2+\mathrm{I}=$ IO

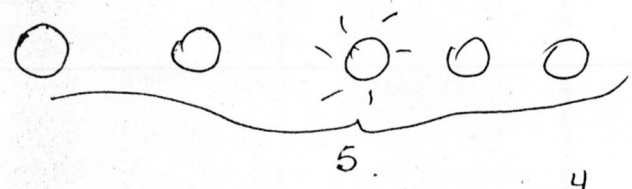

$$
\begin{array}{llllll}
1 & 2 & 3 & 4 & 5 & \begin{array}{r}
2 \\
+0
\end{array} \\
4 & 3 & 2 & 1 & 0 & +0
\end{array}
$$

por lo tanto so son los implecidor.

Poque jo sumo tantas portunidades tengan redaultado de los impleados. 
Estas dos soluciones parciales muestran un pensamiento que se va aproximando a la idea de combinación, pero limitada. Otras respuestas muestran que los alumnos utilizan enfoques combinatorios menos acertados. Uno de los más frecuentes es considerar cinco posibilidades por cada luz y multiplicar $5 \times 5 \times 5 \times 5 \times 5$, dando como resultado 3125 .

Otra opción que aparece en las respuestas es considerar que las combinaciones de 5 tomadas de i en I es 5 elevado a I, las de 5 tomadas de 2 en 2 es 5 elevado a 2 , y así sucesivamente. Al final suma los resultados parciales.

FIGURA 6. Respuesta basada en combinaciones dada por un estudiante

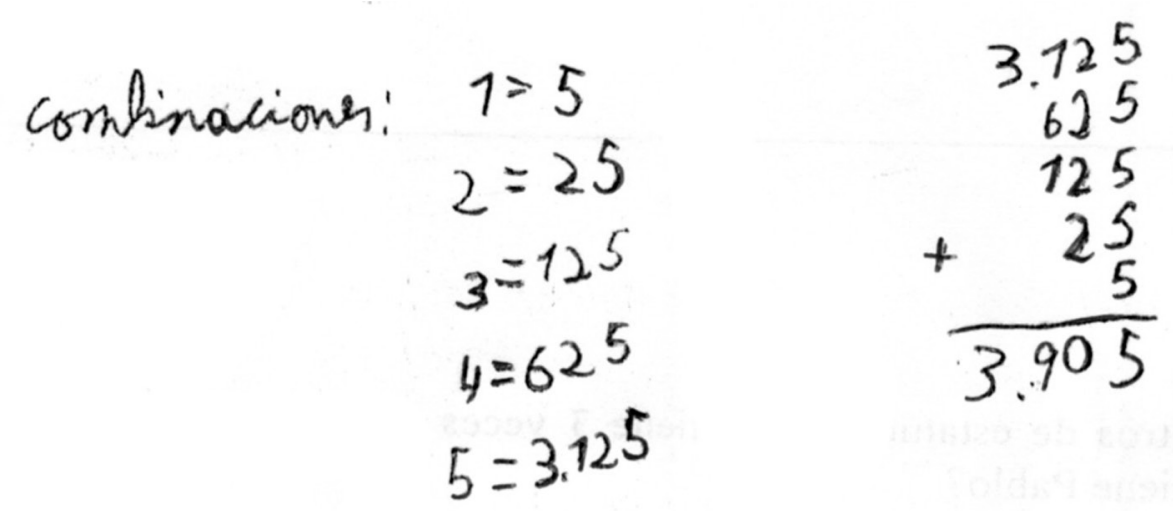

De lo anterior resaltamos que los estudiantes con talento de II y i2 años han empleado las estrategias de la regla del producto que ya habían emergido en los sujetos más jóvenes a la edad de io años. Como novedad hay en ellos un uso incipiente de la idea de combinación, pero limitada a combinaciones de dos elementos. Además se observa que la traducción a modelos matemáticos no es precisa.

Un salto cualitativo en las respuestas se produjo cuando aplicamos este problema en Granada, durante el curso 20II-20I2, a 25 niños del proyecto Estímulo del Talento Matemático-estalmat (Guzmán, 2002a y 2002b), en una de las sesiones en la que era pertinente su empleo. En este caso los niños tenían entre i3 y is años. En el currículo escolar que seguían estos estudiantes, el tema de combinatoria todavía no se había impartido.

El desarrollo de estos estudiantes con talento respecto a estudiantes de menor edad se ha traducido en reproducción de estrategias empleadas por los estudiantes más jóvenes, pero con una mayor calidad de las herramientas matemáticas utilizadas. La regla del producto deja de utilizarse en su forma expandida $2 \times 2 \times 2 \times 2 \times 2$ para reflejarse mayoritariamente en forma $2^{5}$. El mayor progreso en los estudiantes de este rango de edad con respecto a las anteriores descritas, se observa en los estudiantes que utilizan las combinaciones.

a. Diez de los 25 estudiantes utilizan la regla del producto en su forma desarrollada $2 \times 2 \times 2 \times 2 \times 2$ o expresado más escuetamente en la forma $2^{5}$-I. Tres de ellos incorporan el proceso de empezar con casos más simples y generalizar la fórmula obtenida. 
b. Doce estudiantes han procedido a contar el número de combinaciones.

$>$ de I hasta 5 luces y sumar.

D Dibujar todas las combinaciones y contarlas.

c. Dos estudiantes no obtienen la solución.

d. Uno de los estudiantes utiliza la regla del producto, pero se encuentra con un dilema. Por cada una de las cuatro luces primeras empezando por la izquierda, considera que hay dos posibilidades por tanto, $2 \times 2 \times 2 \times 2$, pero para él la quinta no puede estar apagada. Sólo considera la posibilidad de estar encendida. Por tanto da como solución $2^{4} \times$ I.

Las respuestas dadas por los estudiantes del grupo de talento pueden dar pie al profesor a analizar y comparar las dos formas mayoritarias que han empleado los estudiantes con talento:

Como suma de combinaciones de luces: $(\mathrm{I}+5+\mathrm{IO}+\mathrm{IO}+5+\mathrm{I})-\mathrm{I}$

Siguiendo la regla del producto: $2^{5}$ I

Y a formalizar durante un proceso de enseñanza-aprendizaje la igualdad

$$
\left(\begin{array}{l}
5 \\
0
\end{array}\right)+\left(\begin{array}{l}
5 \\
1
\end{array}\right)+\left(\begin{array}{l}
5 \\
2
\end{array}\right)+\left(\begin{array}{l}
5 \\
3
\end{array}\right)+\left(\begin{array}{l}
5 \\
4
\end{array}\right)+\left(\begin{array}{l}
5 \\
5
\end{array}\right)=2^{5}
$$

Que puede generalizarse:

$$
\left(\begin{array}{l}
n \\
0
\end{array}\right)+\left(\begin{array}{c}
n \\
1
\end{array}\right)+\left(\begin{array}{l}
n \\
2
\end{array}\right)+\cdots+\left(\begin{array}{c}
n \\
n-1
\end{array}\right)+\left(\begin{array}{l}
n \\
n
\end{array}\right)=2^{n}
$$

La sesión con alumnos con talento puede continuar conectando estas ideas con el triángulo de Pascal.

\section{Habilidad didáctica para gestionar los retos}

Los profesores deben ser competentes en identificar las posibilidades didácticas de un reto para un grupo de estudiantes y, de acuerdo con ello, seleccionarlo para una determinada labor didáctica. Sobre un mismo objetivo de aprendizaje el profesor puede disponer de distintos retos de mayor o menor complejidad y que, en algún sentido, se complementen desde el punto de vista de su uso didáctico. En este caso el profesor debe utilizar su conocimiento didáctico para gestionar cómo llevar a cabo el desarrollo de la sesión de clase y el papel que jugará cada uno de los retos. Analicemos estas ideas con más detalle.

Los acertijos, juegos de magia y los rompecabezas en general ejercen un gran atractivo para los estudiantes con talento y despiertan su curiosidad. Planteados como retos de aula resultan muy motivadores para los estudiantes, aunque en general son difíciles de resolver. Planteados al inicio de una unidad didáctica, dan pie para introducir y trabajar otros retos menos motivadores, más rutinarios, pero más accesibles 
para el nivel de los estudiantes. Con un segundo reto los estudiantes aprenden una noción o idea matemática que el profesor quiere que los alumnos adquieran durante la realización de la tarea, que subyace también en el reto inicial y que, por tanto, después servirá de modelo a extrapolar y tratar de dar respuesta al reto inicial, bastante más difícil, pero más atractivo para el estudiante.

Como ejemplo de retos complementarios didácticamente abordamos el juego de magia conocido como cartas adivinatorias y el reto que denominamos bolsas de monedas (Department for Education and Employment, 2000). El reto de las cartas adivinatorias se basa en un conjunto de tarjetas (veáse Figura 7) con las que se realiza el truco de adivinarles la edad a los estudiantes. Después de aplicarlo varias veces y adivinar la edad de los estudiantes que se ofrecen voluntarios, se les plantea el reto de si son capaces de encontrar el truco de estas cartas. Y se les anima a intentar encontrar por qué funciona.

Figura 7. Cartas adivinatorias

\section{Cartas adivinatorias}

\begin{tabular}{|r|r|}
\hline \multicolumn{2}{|c|}{ Carta 1 } \\
\hline 1 & 3 \\
\hline 5 & 7 \\
\hline 9 & 11 \\
\hline 13 & 15 \\
\hline 17 & 19 \\
\hline 21 & 23 \\
\hline 25 & 27 \\
\hline 29 & 31 \\
\hline
\end{tabular}

\begin{tabular}{|r|r|}
\hline \multicolumn{2}{|c|}{ Carta 2 } \\
\hline 2 & 3 \\
\hline 6 & 7 \\
\hline 10 & 11 \\
\hline 14 & 15 \\
\hline 18 & 19 \\
\hline 22 & 23 \\
\hline 26 & 27 \\
\hline 30 & 31 \\
\hline
\end{tabular}

\begin{tabular}{|r|r|}
\hline \multicolumn{2}{|c|}{ Carta 3 } \\
\hline 4 & 5 \\
\hline 6 & 7 \\
\hline 12 & 13 \\
\hline 14 & 15 \\
\hline 20 & 21 \\
\hline 22 & 23 \\
\hline 28 & 29 \\
\hline 30 & 31 \\
\hline
\end{tabular}

\begin{tabular}{|r|r|}
\hline \multicolumn{2}{|c|}{ Carta 4 } \\
\hline 8 & 9 \\
\hline 10 & 11 \\
\hline 12 & 13 \\
\hline 14 & 15 \\
\hline 24 & 25 \\
\hline 26 & 27 \\
\hline 28 & 29 \\
\hline 30 & 31 \\
\hline
\end{tabular}

\begin{tabular}{|r|r|}
\hline \multicolumn{2}{|c|}{ Carta 5 } \\
\hline 16 & 17 \\
\hline 18 & 19 \\
\hline 20 & 21 \\
\hline 22 & 23 \\
\hline 24 & 25 \\
\hline 26 & 27 \\
\hline 28 & 29 \\
\hline 30 & 31 \\
\hline
\end{tabular}

Nuestra experiencia personal aplicando en varios cursos sucesivos este reto a alumnos con talento matemático del primer ciclo de secundaria es que no suelen conocer el truco y por supuesto tampoco la razón matemática que subyace en él. También lo hemos aplicado en cursos de formación para maestros egresados, obteniendo el mismo resultado. El reto planteado cumple su función didáctica: motivar e interesar al alumno. La introducción del segundo reto está ya justificada, y aunque el segundo reto sea más rutinario, se aborda con interés.

El profesor aduce a los estudiantes que les va a plantear otro problema similar a este pero más fácil. Y les plantea el nuevo reto, el que hemos denominado bolsas de monedas. En este caso el profesor ha utilizado la estrategia de buscar un reto más sencillo, que contiene el principio subyacente en las tarjetas. El enunciado del mismo es el siguiente:

José reparte is euros entre cuatro bolsas de manera que puede pagar una cantidad en euros comprendida entre i y is euros sin abrir las bolsas. ¿Cuántos euros ha colocado en cada bolsa? 
Figura 8. Reto bolsas de monedas

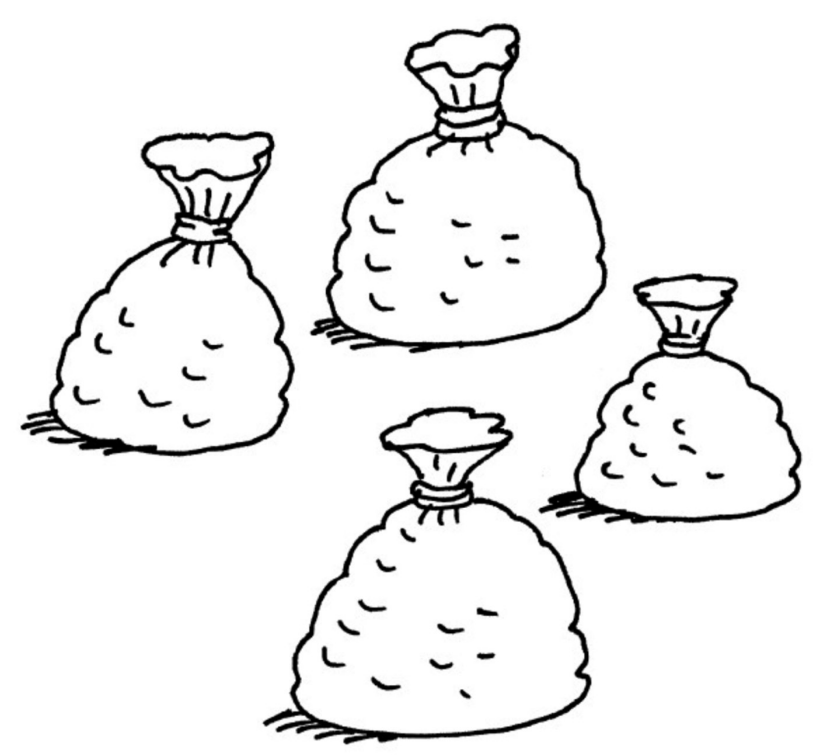

El reto de las bolsas es un reto matemático adecuado para los niños más capaces de $5 .^{\circ}$ y $6 .^{\circ}$ curso de educación primaria (Department for Education and Employment, 2000). El objetivo educativo que le asignan en este documento es que los estudiantes resuelvan problemas y expliquen sus métodos y razonamientos. Es una tarea que suelen asumir fácilmente los estudiantes con talento y los maestros egresados, y es apropiada para poner en práctica la estrategia de ensayo-error y de búsqueda sistemática.

Después de varios ensayos los estudiantes concluyen que una de las bolsas debe contener un euro, puesto que uno no puede expresarse como suma de otros números. A partir de ahí proceden a examinar los números en orden creciente y van descartando los que no proceden. Después de aceptar el uno, razonan que debe haber una bolsa con 2 euros, 3 lo rechazan por ser $\mathrm{I}+2$, aceptan que 4 debe ser el contenido de otra bolsa. Proceden sistemáticamente y encuentran el 8 como el número de euros de la cuarta bolsa.

Llegado a este punto se sistematizan los resultados. El número de euros en las bolsas es:

I, 2, 4 y 8. Y se analizan propiedades que tengan en común estos números, haciendo patente que son las potencias de 2. Los alumnos quedan sorprendidos al descubrir que todo número menor que i6 se puede descomponer como suma de potencias de dos, sin que se repitan ninguna. Esto da pie también a extender estas ideas a números mayores y a la generalización de las mismas: los números menores que $2^{\mathrm{n}}$ se pueden escribir como suma de potencias de dos con exponente menor que $\mathrm{n}$ sin repetir ninguna de ellas.

Si el profesor considera que los estudiantes han satisfecho sus expectativas de aprendizaje, puede utilizar lo aprendido para retomar el reto de las cartas adivinatorias e intentar de nuevo descubrir por analogía que las cartas tienen como número inicial las potencias de dos y que los números aparecen o no en una carta si ese número 
inicial se encuentra en su descomposición como suma de potencias de dos. El número $\mathrm{I} 4=2+4+8$ aparece en las cartas cuya cabecera es 2,4 y 8 .

Para un grupo de niños en una clase inclusiva lo anterior puede ser suficiente, pero en grupos de estudiantes con talento que requieran actividades de enriquecimiento, lo anterior se puede considerar formando parte del aprendizaje del sistema binario de numeración, escribiendo o o I en función de si en la descomposición de un número aparece la potencia de dos correspondiente. Así, I4 $=8+4+2=$ IIIo en base 2 .

El reto de las luces lo puede utilizar el profesor para introducir la escritura de los números binarios. Una vez que los estudiantes han resuelto el problema y vistas las variadas formas que habían empleado, procedemos a obtener la solución por otra vía. Los estudiantes con talento se prestan fácilmente a explorar vías distintas de solución de los problemas. En primer lugar, procedemos a simbolizar las dos posibilidades que tienen cada luz con un o o un I según esté apagada o encendida. Nos situamos en el caso más simple de disponer de dos luces y expresamos todas las posibilidades: oo, oI, Io, II. Pasamos al caso de tres luces y así sucesivamente. Esto es una forma distinta de ver el sistema binario que lo conecta con su empleo en informática.

\section{Conclusión}

Uno de los problemas en las clases de matemáticas de la enseñanza obligatoria es el aburrimiento de los estudiantes. Además de ser un problema generalizado para muchos estudiantes, los alumnos con talento matemático los sufren de manera especial, pues gustan de tareas desafiantes que no suelen incorporarse al currículo escolar en estos niveles educativos. Pasar a los estudiantes a cursos superiores es una de las formas de dar respuesta parcial a este problema, otra alternativa es proporcionarles tareas específicas que los enriquezcan y estimulen su talento. En respuesta a la necesidad de estimular y potenciar el talento de los estudiantes más capaces, han surgido programas extraescolares como ESTALMAT (Guzmán, 2002a y 2002b) que tratan de suplir las limitaciones que impone una enseñanza unificada para estudiantes de la misma edad pero con distintas capacidades. Estos programas alcanzan sólo a una pequeña parte de la población de los niños con talento matemático, por lo que hay que imaginar formas de superar la enseñanza unificada en las clases de matemáticas que permitan incorporar las ideas que presiden estos programas de estímulo del talento.

En este trabajo, consideramos que los retos y los desafíos matemáticos deben formar parte de las tareas escolares destinadas al enriquecimiento de los estudiantes con talento. El profesor de matemáticas tiene en este empeño la función especial de seleccionar y adecuar las tareas a las distintas capacidades de los estudiantes. Las ideas que hemos desarrollado en este trabajo les pueden ser útiles a los profesores y orientarlos en este sentido.

\section{Bibliografía}

Applebaum, M. y Leikin, R. (2007) Teachers' conceptions of mathematical challenge in school mathematics. En J. W. Woo, H. C. Lew, K. S. Park y D. Y. Seo (eds.) Proceedings of the 3Ist Conference of the International Group for the Psychology of Mathematics Education, vol. 2 (pp. 9-16). Seoul, Korea: PME. 
RETOS, PROFESORES Y ALUMNOS CON TALENTO MATEMÁTICO

Assouline, S. G. y Lupkowski-Shoplik, A. (2005) Developing math talent: A guide for educating gifted and advanced learners in math. Waco, Tx: Prufrock Press.

Barbeau, E. J. y Taylor, P. J. (eds.) (2009) Challenging Mathematics In and Beyond the Classroom. The I6th ICMI Study. New York: Springer.

Benavides, M. (2008) Caracterización de sujetos con talento en resolución de problemas de estructura multiplicativa. Tesis doctoral. Universidad de Granada. Granada.

Castro, E. (1995) Niveles de comprensión en problemas verbales de comparación multiplicativa. Granada: Comares.

Castro, E.; Benavides, M. y Segovia, I. (2006) Cuestionario para caracterizar a niños con talento en resolución de problemas de estructura multiplicativa. Faisca. Revista de Altas Capacidades, $\mathrm{I} 3,4^{-22}$.

Charles, R. y Lester, F. (1982) Teaching Problem Solving. What, Why y How. Palo Alto, ca: Dale Seymour Publications.

Collins, A.; Brown, J. S. y Newman, S. E. (1989) Cognitive apprenticeship: Teaching the crafts of reading, writing, and mathematics. En L. B. Resnick (ed.) Knowing, learning, and instruction: Essays in honor of Robert Glaser (pp. 453-494). Hillsdale, NJ: Erlbaum.

Department For Education and Employment (2000) Mathematical challenges for able pupils. London (El autor).

DoyLe, W. (1988) Work in mathematics classes: The context of students' thinking during instruction. Educational Psychologist, 23 (2), I67-180. http://dx.doi.org/IO.I207/si5326985ep2302_6

Ellerton, N. (1986) Children's Made-Up Mathematics Problems - A New Perspective on Talented Mathematicians. Educational Studies in Mathematics, 17, 26I-27I. http://dx.doi.org/I0.1007/BFo0305073

Feldhusen, J. F. y Kroll, M. D. (I99i) Boredom or challenge for the academically talented in school. Gifted Education International, 7, 80-81. http://dx.doi.org/I0.1177/026142949100700207

Freiman, V. (2006) Problems to discover and to boost mathematical talent in early grades: a challenging situations approach. The Montana Mathematics Enthusiast, 3, 5I-75.

Greenes, C. (198I) Identifying the gifted student in mathematics. Aritmetic Teacher, 28 (8), I4-I7.

Guzmán, M. (2002a) Una descripción del proyecto «Detección y estímulo del talento matemático precoz en la Comunidad de Madrid». Bordón. Revista de Pedagogía, 54, 255-268.

Guzmán, M. (2002b) Un programa para detectar y estimular el talento matemático precoz en la Comunidad de Madrid. Gaceta de la Real Sociedad Matemática Española, 5 (I), I3I-I44.

Henningsen, M. y Stein, M. K. (1997) Mathematical tasks and student cognition: classroombased factors that supports and inhibit high-level mathematical thinking and reasoning. Journal for Research in Mathematics Education, 28 (5), 524-549. http://dx.doi.org/Io.2307/749690

Hiebert, J.; Carpenter, T.; Fennema, E.; Fuson, K.; Wearne, D.; Murray, H.; Olivier, A. y Human, P. (1997) Making Sense. Portsmouth, NH: Heinemann.

Johnson, D. T. (1994) Mathematics curriculum for the gifted. En J. VanTassel-Baska (ed.) Comprehensive curriculum for gifted learners. Boston: Allyn and Bacon.

KrUteTsKiI, V. A. (1969) An analysis of the individual structure of mathematical abilities in schoolchildren. En J. Kilpatrick e I. Wirszup (eds.) Soviet Studies in the Psychology of Learning and Teaching Mathematics, vol. II (pp. 59-I04). The Structure of Mathematical Abilities. Chicago: University of Chicago Press.

LAMPERT, M. (1990) When the problem is not the question and the solution is not the answer: Mathematical knowing and teaching. American Educational Research Journal, 27 (I), 29-63. http://dx.doi.org/I0.3102/00028312027001029

Leikin, R. (2004) Towards High Quality Geometrical Tasks: Reformulation of a Proof Problem. En M. J. Hoines y A. B. Fuglestad (eds.) Proceedings of the $28^{\text {th }}$ International Conference for the Psychology of Mathematics Education, vol. 3 (pp. 209-216). 
Miller, R. C. (1990) Discovering Mathematical Talent. ERIC Digest \#- 482.

Niederer, K. e Irwin, K. (20OI) Using problem solving to identify mathematically gifted Students. En M. van Den Heuvel-Panhuizen (ed.) Proceeding of the 25 th Conference of the International Group for the Psychology of Mathematics Education, vol. 3 (pp. 43I-438). Utrecht.

Niederer, K.; Irwin, R. C.; Irwin, K. C. y Reilly, I. L. (2003) Identification of Mathematically Gifted Children in New Zealand. High Ability Studies, I4 (I), 7I-84. http://dx.doi.org/10.1080/13598130304088

Pasarín, M. J.; Feijoo, M.; Díaz, O. y Rodríguez, L. (2004) Evaluación del talento matemático en educación secundaria. Faisca. Revista de Altas Capacidades, II, 88-ıо3.

Polya, G. (1973). Cómo plantear y resolver problemas. México: Trillas.

Powell, A. B.; Borge, I. C.; Fioriti, G. I.; Kondratieva, M.; Koublanova, E. y Sukthankar, N. (2009) Challenging tasks and mathematics learning. En Challenging Mathematics In and Beyond the Classroom (pp. 133-170). Springer us.

Schoenfeld, A. H. (1985) Mathematical problem solving. Orlando, Fl: Academic Press.

SHEFFIELD, L. J. (ed.) (1999) Developing mathematically promising students. Reston, va: National Council of Teachers of Mathematics.

ShefField, L. J. (2003) Extending the Challenge in Mathematics: Developing Mathematical Promise in K-8 Students. Thousand Oaks, ca: Corwin Press.

Span, P. y Overtoom-Corsmit, R. (1986) Information Processing by Intellectually Gifted Pupils Solving Mathematical Problems. Educational Studies in Mathematics, 17, 273-295. http://dx.doi.org/I0.1007/BFo0305074

Watters, J. J. y Diezmann, C. M. (2000) Catering for mathematically gifted elementary students: Learning from challenging tasks. Gifted Child Today, 23 (4), I4-19. 